\title{
Upright posture and maximal exercise increase platelet aggregability and prostacyclin production in healthy male subjects
}

\author{
DaLi Feng, Jaime Murillo, Praveen Jadhav, Carol McKenna, Otavio C Gebara, \\ Izabella Lipinska, James E Muller, Geoffrey H Tofler
}

\begin{abstract}
Background-It is well accepted that heavy physical exertion can trigger the onset of myocardial infarction, but the mechanism is uncertain. As platelet and endothelial function play an important role in thrombotic events, platelet and prostacyclin responses to maximal treadmill exercise were studied.

Methods/Results-The study subjects were 40 healthy men, mean (SEM) age 29 (5) years. Platelet aggregation was measured on a four channel aggregometer. Plasma 6-keto-prostaglandin $\mathbf{F}_{1 \alpha}$ was analysed using an enzyme immunoassay technique. Upright posture and exercise produced an increase in platelet aggregability, as indicated by a fall in the threshold concentration of adrenaline (epinephrine) from 7.6 (1.5) $\mu M$ at rest to 4.3 (1.0) $\mu M$ after exercise $(p=0.002)$. The collagen lag time became significantly shorter with exercise (from 79.1 (3.1) seconds at rest to 71.9 (2.6) seconds after exercise, $p=0.003$ ). Exercise was also associated with a $55 \%$ increase in plasma 6-keto-prostaglandin $\mathbf{F}_{1 \alpha}$ (from $38.1(75 \%$ CI 29.0 to 46.5$) \mathrm{pg} / \mathrm{ml}$ at rest to $59.2(47.3$ to 66.8$) \mathrm{pg} / \mathrm{ml}$ after exercise, p<0.001).

Conclusions-In healthy male subjects, upright posture and maximal exercise increased platelet aggregability but this increase was counteracted by an increase in prostacyclin production. In patients with endothelial dysfunction, a reduced prostacyclin response to exercise may promote a transient prothrombotic imbalance that may trigger cardiovascular disease onset. (Br F Sports Med 1999;33:401-404)
\end{abstract}

Keywords: exercise; platelet; collagen; 6-ketoprostaglandin $\mathrm{F}_{1 \alpha}$; cardiovascular disease

Heavy physical exertion has been shown to trigger the onset of acute myocardial infarction and sudden cardiac death, particularly in sedentary people. ${ }^{12}$ Platelet activation plays an important role in the pathogenesis of these acute coronary ischaemic syndromes. Firstly, myocardial infarction and sudden cardiac death are often associated with platelet rich thrombi or platelet aggregates. ${ }^{3}$ Secondly, biochemical markers of platelet activation are increased during episodes of acute cardiac ischaemia. ${ }^{4}$ Finally, antiplatelet agents have been shown to reduce the incidence of cardiovascular ischaemic events.
Stress induced hypercoagulability may be a link between a stressor and an acute coronary event. $^{5}$ The effect of exercise on platelet aggregability remains uncertain, with reports indicating an increase, ${ }^{6-12}$ no change, ${ }^{13-16}$ or even a decrease ${ }^{17}$ in platelet reactivity. The effect of exercise on platelet adhesion is also poorly defined, in part because of the use of methodology that could not reliably distinguish platelet adhesion from aggregation in those studies. ${ }^{6718}$

We therefore studied 40 healthy men to determine the effect of maximal treadmill exercise on platelet aggregability and adhesion. In addition, we also determined the effect of exercise on thromboxane and prostacyclin production.

\section{Methods}

STUDY POPULATION

The study protocol was approved by the institutional review board of the Deaconess Hospital. The investigation was in accordance with the Declaration of Helsinki. Informed consent was obtained from all subjects. Exclusion criteria and subject characteristics have been described in detail previously. ${ }^{19}$ The data reported here are from responses observed in the placebo phase of a study of the effect of aspirin dosage and enteric coating on platelet reactivity. Whereas the preceding paper focused on the effect of aspirin on platelet aggregability and prostacylin, this one provides information on the effect of exercise on platelet reactivity. ${ }^{19}$

Forty healthy untrained men completed the study. The mean (SEM) age was 29 (5) years. At screening, a medical history was taken and subjects underwent a physical examination, electrocardiogram, and blood drawing for platelet aggregation studies, blood count, and biochemistry profile. Those who met enrolment criteria returned within two weeks of screening for study. After a standardised breakfast, subjects rested for 30 minutes in the supine position and then performed a maximal exercise treadmill test using the Bruce protocol. Blood samples were obtained immediately from an antecubital vein before and after exercise while the subjects were in the supine position. Blood was withdrawn in a syringe containing $3.8 \%$ sodium citrate $(9: 1, v / v)$. Platelet rich plasma was separated immediately after blood withdrawal by centrifugation for 10 minutes at $160 \mathrm{~g}$.

PLATELET AGGREGATION

Platelet aggregation was measured on a four channel aggregometer at $37^{\circ} \mathrm{C}$ by the method of Born..$^{20}$ The aggregating agents tested were adrenaline (epinephrine) and ADP at various
Accepted for publication 3 June 1999 
concentrations $(0.01-30 \mu \mathrm{M})$ and a fixed concentration of collagen $(0.19 \mathrm{mg} / \mathrm{ml})$. At each time point, the following parameters were obtained: (a) the lowest concentration of adrenaline and ADP required to produce a biphasic response with greater than 50\% aggregation (threshold concentration); $(b)$ the collagen lag time, defined as the time interval in seconds from the addition of $0.19 \mathrm{mg} / \mathrm{ml}$ collagen to the commencement of aggregation.

PLATELET ADHESION

Platelet adhesion was measured using a parallel plate flow chamber in which platelet rich plasma was perfused at a constant flow rate to obtain a shear stress of $26 \mathrm{~s}^{-1}$. The modified Hele-Shaw chamber consisted of a glass slide coated with fibronectin, attached to the flow chamber by a perimeter vacuum pump. ${ }^{21}$ The apparatus was mounted on an Olympus inverted microscope equipped with Hoffman Contrast. The adhesion process was recorded by taking photographs of the coated surface every 30 seconds. The total number of platelets adherent to the slide was counted after $10 \mathrm{~min}$ utes of flow. Platelet analysis was completed within two hours of the blood collection.

ASSAY OF PLASMA 6-KETO-PROSTAGLANDIN $\mathrm{F}_{1 \alpha}$ AND THROMBOXANE B

Blood samples were withdrawn and mixed immediately with citrate/citric/dextrose, containing $20 \mu \mathrm{M}$ indomethacin in a ratio of blood to anticoagulant of $6: 1$. The blood samples were then placed on ice and centrifuged at $2000 \mathrm{~g}$ at $4^{\circ} \mathrm{C}$ for 15 minutes. The platelet poor plasma was removed and frozen at $-80^{\circ} \mathrm{C}$ for later analysis using an enzyme immunoassay technique. $^{22}$ The interassay and intra-assay coefficients of variation were $9.5 \%$ and $7.2 \%$ for 6-keto-prostaglandin $\mathrm{F}_{1 \alpha}$ and $8.6 \%$ and $5.6 \%$ for thromboxane $\mathrm{B}_{2}$.

STATISTICAL ANALYSIS

The paired $t$ test, or Wilcoxon Signed Rank test when the variables were not normally distributed, were used to compare results before and after exercise. All data are reported as mean (SEM) except for 6-keto-prostaglandin $\mathrm{F}_{1 \alpha}$ and thromboxane $\mathrm{B}_{2}$ levels, which are expressed as median ( $75 \%$ confidence interval) because the distribution was abnormal. A two tailed $\mathrm{p}$ value $<0.05$ was regarded as statistically significant. Statistical analysis was performed using Sigma Stat software. ${ }^{23}$

\section{Results}

The mean (SEM) exercise duration in these healthy subjects was 13.5 (0.3) minutes. Heart rate increased significantly from 78 (2) beats per minute at baseline to a peak of 189 (1) $(\mathrm{p}<0.0001)$. Systolic arterial pressure also increased from 117 (2) $\mathrm{mm} \mathrm{Hg}$ at baseline to 169 (2) $\mathrm{mm} \mathrm{Hg}$ at peak exercise $(\mathrm{p}<0.0001)$. Diastolic blood pressure decreased with exercise from 77 (1) at baseline to 73 (1) after exercise $(\mathrm{p}=0.0009)$.

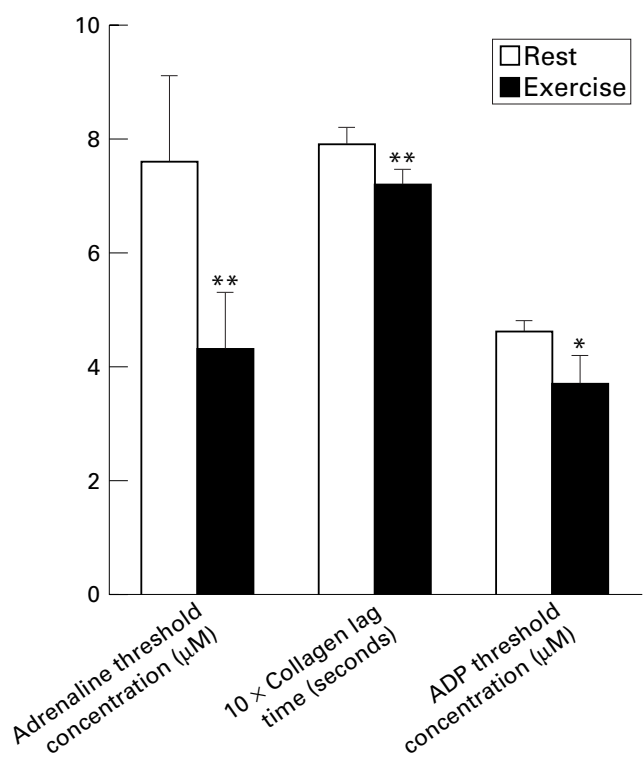

Figure 1 Platelet aggregability induced by adrenaline, collagen, and ADP at rest and immediately after maximal exercise. ${ }^{\star} p=0.06,{ }^{\star} p<0.01$ compared with value at rest.

PLATELET AGGREGABILITY (FIG 1)

Adrenaline induced aggregation

Exercise significantly increased platelet aggregability as indicated by a reduction in the adrenaline threshold concentration from 7.6 (1.5) $\mu \mathrm{M}$ at rest to $4.3(1.0) \mu \mathrm{M}$ after exercise $(\mathrm{p}=0.002)$.

\section{Collagen lag time}

The mean collagen lag time shortened significantly with exercise from 79.1 (3.1) seconds at rest to 71.9 (2.6) seconds after exercise ( $\mathrm{p}=$ 0.003 ). The shortening of the collagen lag time indicates an increase in platelet aggregability.

\section{ADP induced aggregation}

There was a trend toward reduction of ADP threshold concentration during exercise. Threshold concentrations were $4.6(0.2) \mu \mathrm{M}$ at rest $v 3.7(0.5) \mu \mathrm{M}$ after exercise $(\mathrm{p}=0.06)$.

\section{Platelet count}

The platelet count in platelet rich plasma increased significantly after exercise, from $201000(4000) / \mu 1$ at rest to $236000(7000) / \mu 1$ after exercise $(p<0.0001)$. Neither the absolute platelet count nor the exercise induced increase from baseline correlated significantly with changes in adrenaline, collagen, or ADP induced aggregability ( $\mathrm{p}$ values all $>0.05$ ).

\section{PLATELET ADHESION}

The number of platelets adherent to a fibronectin slide after 10 minutes of perfusion was not altered by exercise $(123(14)$ at rest $v$ 117 (13) after exercise, $\mathrm{p}=0.40$ ).

PLASMA THROMBOXANE $\mathrm{B}_{2}$ (TABLE 1)

Exercise tended to increase plasma thromboxane production, from 54.6 (36.6 to 81.7 ) $\mathrm{pg} / \mathrm{ml}$ at rest to 73.5 (42.8 to 93.4$) \mathrm{pg} / \mathrm{ml}$ after exercise $(\mathrm{p}=0.15)$. 
Table 1 Plasma prostacyclin and thromboxane levels ( $\mathrm{pg} / \mathrm{ml})$

\begin{tabular}{llll}
\hline & Rest & Exercise & p value \\
\hline 6-Keto-prostaglandin $\mathrm{F}_{1 \alpha}$ & $38.1(29.0$ to 46.5$)$ & $59.2(47.3$ to 66.8$)$ & $<0.001$ \\
Thromboxane $\mathrm{B}_{2}$ & $54.6(36.6$ to 81.7$)$ & $73.5(42.8$ to 93.4$)$ & 0.15
\end{tabular}

Values are mean $(75 \%$ confidence interval).

PLASMA 6-KETO-PROSTAGLANDIN $\mathrm{F}_{1 \alpha}$ (TABLE 1) Exercise was associated with a $55 \%$ increase in plasma 6-keto-prostaglandin $\mathrm{F}_{1 \alpha}$ (38.1 (29.0 to $46.5) \mathrm{pg} / \mathrm{ml}$ at rest $v 59.2(47.3$ to 66.8$) \mathrm{pg} / \mathrm{ml}$ after exercise, $\mathrm{p}<0.001)$.

\section{Discussion}

In this study of healthy men, upright posture and maximal treadmill exercise significantly increased the platelet reactivity to adrenaline and collagen induced aggregation, thereby creating a prothrombotic change. However, exercise in these healthy subjects also produced a counteracting antithrombotic increase in prostacyclin production.

Although regular physical activity is protective against cardiovascular disease, several epidemiological studies indicate that heavy exertion, particularly in sedentary people, may trigger acute myocardial infarction and sudden cardiac death. ${ }^{12}$ The increased incidence of myocardial infarction and sudden cardiac death in the morning also coincides with increased sympathoadrenal activity and increased platelet aggregability. ${ }^{24}$ Stress induced platelet activation may be a link between exertion and an acute coronary event. ${ }^{5}$

\section{EFFECT OF MAXIMAL EXERCISE ON PLATELET} AGGREGABILITY

The effect of exercise on platelet aggregability is controversial, with varying results being obtained. This variation is due in part to differences in exercise protocol, subject characteristics, or method of assessment of platelet activation. In most, but not all, studies, short strenuous exercise has been found to increase platelet aggregability, ${ }^{679112526}$ whereas mild or moderate exercise tends to have no effect on platelet reactivity or may even decrease it. ${ }^{14} 142526$ Chicharro et $a l^{25}$ and Wang et $a l^{26}$ evaluated the relation between exercise intensity and platelet aggregability. They found that only strenuous exercise or exercise to an anaerobic threshold resulted in an increase in platelet aggregability. The type of exercise does not seem to influence the platelet aggregability response. ${ }^{6-12} 25-27$ The characteristics of the study subjects may also modify the platelet response to exercise. In subjects who exercise regularly, platelet reactivity tends to be unchanged by exercise, whereas in sedentary subjects, exercise results in a heightened platelet response. $^{1011162628}$ Sinzinger and Fitscha ${ }^{10}$ and Wang et $a l^{27}$ reported that the enhanced platelet aggregability produced by short term vigorous exercise can be reduced by exercise training. Furthermore, aggregability returned to the higher pretraining levels after deconditioning. ${ }^{27}$ These findings may help to explain the paradox between the triggering effect of heavy exertion and the protective effect of regular moderate exertion. ${ }^{1}$
Correction for changes in platelet count modified the effect on platelet aggregability. Whereas studies that do not correct for the increased platelet count after exercise tend to show an increased aggregability, ${ }^{6-9} 122627$ those that use a standardised platelet count mostly show no change. ${ }^{12}{ }^{1428}$ In our study, neither the absolute platelet count nor the change in count with exercise correlated with platelet aggregability, suggesting that the increased platelet number with exercise is not the sole mechanism of the observed increase in platelet aggregability.

Recently, using fluorescence labelled monoclonal antibodies to detect activation dependent platelet surface receptor changes, Kestin and colleagues ${ }^{29}$ found an increase in platelet activity after strenuous exercise in sedentary healthy subjects. Their results are consistent with our study. The mechanism of the platelet activating effect of physical exercise is unclear. Several mechanisms are probably involved, such as catecholamine stimulation, enhanced thrombin generation, and shear induced aggregation resulting from increased cardiac output and blood flow.

Posture change has been shown to have a significant effect on platelet aggregability. ${ }^{30} 31$ As exercise was performed in the upright posture, we cannot exclude the possibility that the increase in platelet aggregability was due in part to assumption of this posture. ${ }^{30}{ }^{31}$ However, blood samples were obtained in the supine position both before and after exercise. We also did not have detailed information on the fitness levels of the subjects, which may influence platelet aggregation.

\section{EFFECT OF MAXIMAL EXERCISE ON PLATELET} ADHESION

In contrast with platelet aggregability, platelet adhesion as assessed by fibronectin adherence was not influenced by maximal exercise in our study. Several static and dynamic systems have been used to measure platelet adhesion. These include the use of glass beads, a rotating glass bulb, and a glass-wool filter technique. ${ }^{6} 7183233$ With these methods, platelet adhesiveness has been variably reported to be increased, ${ }^{7} 1832$ unchanged, ${ }^{6}$ or even decreased ${ }^{33}$ by exercise. As the assays do not accurately distinguish platelet adhesion from aggregation, the conclusions drawn from the studies are uncertain. In our modified Hele-Shaw flow chamber system, we were able to accurately monitor and quantify platelet adhesion to a fibronectin coated surface.

In contrast with our results, Wang and colleagues $^{26}$, using a similar parallel plate chamber system, found an increase in platelet adhesion with strenuous exercise. However, there are several methodological differences between their system and ours. Firstly, in their static chamber system, platelet rich plasma was gently infused into the chamber and kept there for 12 minutes to allow platelets to settle on the fibrinogen coated surface. The chamber was then flushed with Tyrode's/Hepes buffer for five minutes. In contrast, we continuously perfused platelet rich plasma at a shear rate of 26 $\mathrm{s}^{-1}$ and dynamically monitored platelet adhesion as it might occur on a plaque exposed to 
blood flow. Secondly, as the index of platelet adhesion, they used the slope of attached platelets versus shear stress, whereas we used the absolute number of platelets that adhered to the slide. Thirdly, they used fibrinogen as a coating material whereas we used fibronectin.

MAXIMAL EXERCISE AND PROSTACYCLIN

PRODUCTION

In our study, prostacyclin levels significantly increased with maximal exercise. Prostacyclin is a potent vasodilator and platelet inhibitor. This increase in prostacyclin, as has been shown by other investigators, ${ }^{34-38}$ would tend to balance any prothrombotic tendency induced by strenuous exercise. In addition, strenuous exercise has been shown to significantly increase fibrinolytic activity, ${ }^{71} 173032$ which can also protect against thrombosis. However, these protective responses may be attenuated in subjects with coronary heart disease who show reduced prostacyclin release $e^{35} 38$ or fibrinolytic response $\mathrm{s}^{40-42}$ and greater thromboxane production after stress. ${ }^{35} 38{ }^{39}$ In the presence of a ruptured plaque and collagen exposed to flowing blood, the exercise induced increase in platelet aggregability in the presence of reduced prostacyclin release may promote occlusive thrombosis and acute myocardial infarction. ${ }^{1}$

This study was supported by a grant from Smith-Kline Beecham.

1 Mittleman MA, Maclure M, Tofler GH, et al. Triggering of acute myocardial infarction by heavy physical exertion: protection against triggering by regular exertion. $N$ Engl f Med 1993;329:1677-83.

2 Siscovic DS, Weiss NS, Fletcher RH, et al. The incidence of primary cardiac arrest during vigorous exercise. $N$ Engl $f$ Med 1984;311:874-7.

3 Davies MJ, Thomas AC, Knapman PA, et al. Intramyocardial platelet aggregation in patients with unstable angina suffering sudden ischemic cardiac death. Circulation 1986 73:418-27.

4 Fitzgerald DJ, Roy L, Catella F, et al. Platelet activation in unstable coronary disease. $N$ Engl $\mathcal{F}$ Med 1986;315:983-9.

5 Muller JE, Tofler GH, Stone PH. Circadian variation and triggers of onset of acute cardiovascular disease. Circulation 1989;7:733-43

6 Warlow CP, Ogston D. Effect of exercise on platelet count, adhesion and aggregation. Acta Haematol 1974;52:47-52.

7 Prentice CRM, Hassanein AA, McNicol GP, et al. Studies on blood coagulation, fibrinolysis, and platelet function after exercise in normal and splenectomised people. $B r \mathcal{F}$ Haematol 1972;23:541-52.

8 Dimitriadou C, Dessypris A, Louisou C, et al. Marathon run II: Effects on platelet aggregation. Thromb Haemost 1977;37:451-5.

9 Poller L, Priest CM, Thomson JM. Platelet aggregation and strenuous exercise. F Physiol (Lond) 1971;213:525-31.

10 Sinzinger H, Fitscha P. Jogging causes a significant increase in platelet sensitivity to prostacyclin. Int $\mathcal{F}$ Sports Med 1986; 7:338-41.

11 Piret A, Niset G, Depiesse E, et al. Increased platelet aggregability and prostacyclin biosynthesis induced by intense exercise. Thromb Res 1990;57:685-95.

12 Hendra TJ, Oughton J, Smith CT, et al. Exercise induced changes in platelet aggregation: a comparison of whole blood and platelet rich plasma techniques. Thromb Res 1988;52:443-51.

13 Vind J, Gleerup G, Nielsen PT, et al. The impact of static work on fibrinolysis and platelet function. Thromb Res 1993;72:441-6.

14 Burghuber O, Sinzinger H, Silberbauer K, et al. Decreased prostacyclin sensitivity of human platelets after jogging and
squash. Prostaglandins and Medicine 1981;6:127-30.
15 Small M, Tweddel AC, Rankin AC, et al. Blood coagulation and platelet function after maximal exercise: effects of beta-adrenoceptor blockade. Haemostasis 1984,14:262-8.

16 Mandalaki T, Dessypris A, Louisou C, et al. Marathon run III: Effects on coagulation, fibrinolysis, platelet aggregation and serum cortisol levels. Thromb Haemost 1977;43:49-52.
Dag B, Gllerup G, Bak AM, et al. Effect of supine exercise on platelet aggregation and fibrinolytic activity. Clin Physiol 1994;14:181-6.

18 Finkel A, Cumming GR. Effects of exercise in the cold on blood clotting and platelets. F Appl Physiol 1965;20:423-4.

19 Feng DL, McKenna C, Murillo J, et al. Effect of aspirin dosage and enteric-coating on platelet reactivity. Am $\mathcal{f}$ Cardiol 1997;80:189-93.

20 Born GVR. Aggregation of blood platelets by adenosine diphosphate and its reversal. Nature 1967;194:927-9.

21 Richardson PD, Mohammed SF, Mason RG. Flow chamber studies of platelet adhesion at controlled spatially varied shear rates. Proceedings of the European Society for Artificial Organs 1977;4:175-88.

22 Pradelles P, Grassi J, Maclouf J. Enzyme immunoassays of eicosanoids using acetylcholine asterase as label: an alternative to radioimmunoassay. Anal Chem 1985;57:1170-3.

23 Jandel Scientific. Comparing repeated measurement of the same individuals. In: The SigmaStat statistical software user's manual. San Rafael, CA: SigmaStat, 1994;8:1-76.

24 Tofler GH, Brezinski D, Schafer AI, et al. Concurrent increase in platelet aggregability and the risk of myocardial infarction and sudden cardiac death. $N$ Engl $\mathcal{f}$ Med 1987;316:1514-18.

25 Chicharro JL, Sanchez O, Bandres F, et al. Platelet aggregability in relation to the anaerobic threshold. Thromb Res 1994;75:251-7

26 Wang JS, Jen CY, Kung HC, et al. Different effects of strenuous exercise and moderate exercise on platelet function in men. Circulation 1994;90:2877-85.

27 Wang JS, Jen CY, Chen HI. Effects of exercise training and deconditioning on platelet function in men. Arterioscler Thromb Vasc Biol 1995;15:1668-74.

28 Siess W, Lorenz R, Roth P, et al. Plasma catecholamines, platelet aggregation and associated thromboxane formation after physical exercise, smoking or norepinephrine infusion. Circulation 1982;66:44-8.

29 Kestin AS, Ellis PA, Barnard MR, et al. Effect of strenuous exercise on platelet activation state and reactivity. Circulation 1993;88:1502-11.

30 Winther K, Hillegas W, Tofler GH, et al. Effects on platelet aggregation and fibrinolytic activity during upright posture and exercise in healthy men. Am f Cardiol 1992;70:1051-5.

31 Brezinski DA, Tofler GH, Muller JE, et al. Morning increase in platelet aggregability: association with assumption of the upright posture. Circulation 1988;78:35-40.

32 Hawkey CM, Britton BJ, Wood WG, et al. Changes in blood catecholamine levels and blood coagulation and fibrinolytic activity in response to graded exercise in man. Br f Haematol 1975;29:377-84.

33 Pegrum GD, Harrison KM, Shaw S, et al. Effect of prolonged exercise on platelet adhesiveness. Nature 1967; 213:301-2.

34 Boger RH, Bode-Boger SM, Schroder EP, et al. Increased prostacyclin production during exercise in untrained and trained men: effect of low-dose aspirin. F Appl Physiol 1995;78:1832-8.

35 Mehta J, Mehta P, Horalek C. The significance of platelet-vessel wall prostaglandin equilibrium during exercise-induced stress. Am Heart f 1983;105:895-900.

36 Wennmalm A, Edlund A, Sevastic B, et al. Excretion of thrombosxane A2 and prostacyclin metabolites during treadmill exercise in patients with intermittent claudication. Clin Physiol 1988;8:243-5.

37 Koivisto VA, Jantunen M, Sane T, et al. Stimulation of prostacyclin synthesis by physical exercise in type I diabetes. Diabetes Care 1989;12:609-14.

38 Wennmalm A, Nowak J, Bjuro T. Excretion of thromboxane A2 and prostacyclin metabolites before and after exercise testing in patients with and without signs of ischemic heart disease. Circulation 1990;82:1737-43.

39 Kishi Y, Ashikaga T, Numano F. Inhibition of platelet aggregation by prostacyclin is attenuated after exercise in patients with angina pectoris. Am Heart f 1992;123:291.

40 Estelles A, Tormo G, Aznar J, et al. Reduced fibrinolytic activity in coronary heart disease in basal conditions and after exercise. Thromb Res 1985;40:373-83.

41 Aznar J, Estelles A, Tormo G, et al. Plasminogen activator inhibitor activity and other fibrinolytic variables in patients with coronary artery disease. Br Heart 7 1988;59:535-41.

42 Drygas WK, Rocker L, Boldt F, et al. The hemostatic and fibrinolytic system in normal subjects and myocardial infarct patients. Effect of a standardised aerobic and inforic and 1987;112:995-9.

\section{Take home message}

In healthy subjects, maximal exercise increases platelet aggregability, but this increase is counteracted by an increase in prostacyclin. In patients with endothelial dysfunction, a reduced prostacyclin response to exercise may promote a transient prothrombotic imbalance that may trigger cardiovascular disease onset. 